\title{
Finances solidaires informelles au Pérou dans la perspective
} du capital social

Informal solidarity finance in Peru from a social capital perspective

\section{Finanzas solidarias informales en Perú desde la perspectiva del capital social}

\section{Sonia Tello-Rozas}

Numéro 337, juillet 2015

L’ESS en Amérique latine : de nouvelles pratiques

New Practices in the Social and Solidarity Economy in Latin America

URI : https://id.erudit.org/iderudit/1032526ar

DOI : https://doi.org/10.7202/1032526ar

Aller au sommaire du numéro

\section{Éditeur(s)}

Association Recma

ISSN

1626-1682 (imprimé)

2261-2599 (numérique)

Découvrir la revue

Citer cet article

Tello-Rozas, S. (2015). Finances solidaires informelles au Pérou dans la perspective du capital social. Revue internationale de l'économie sociale, (337), 100-112. https://doi.org/10.7202/1032526ar
Résumé de l'article

Cette recherche explore les raisons qui motivent la participation aux finances solidaires informelles dans le contexte de l'Amérique latine, celui du Pérou notamment, à travers la notion de capital social. Dans ce but, nous examinons le cas des associations d'épargne et de crédit rotatif (AECR), une forme de crédit tontinier fondée sur la confiance et l'information partagée. Malgré le développement et la consolidation des microfinances dans ce pays, on observe que les associations de type tontinier sont encore très utilisées. A partir d'une étude exploratoire qualitative, nos résultats suggèrent que le type de capital social mobilisé explique en partie les motifs de leur utilisation. Par ailleurs, notre analyse met en exergue les motivations sociales, moins souvent explorées dans la littérature. 


\title{
FINANCES SOLIDAIRES INFORMELLES AU PÉROU DANS LA PERSPECTIVE DU CAPITAL SOCIAL
}

\author{
par Sonia Tello-Rozas*
}

\begin{abstract}
* Ecole des sciences de la gestion (ESG), université de Québec à Montréal (Uqam). Mél. : tello-rozas.sonia@ uqam.ca.
\end{abstract}

Cette recherche explore les raisons qui motivent la participation aux finances solidaires informelles dans le contexte de l'Amérique latine, celui du Pérou notamment, à travers la notion de capital social. Dans ce but, nous examinons le cas des associations d'épargne et de crédit rotatif(AECR), une forme de crédit tontinier fondée sur la confiance et l'information partagée. Malgré le développement et la consolidation des microfinances dans ce pays, on observe que les associations de type tontinier sont encore très utilisées. A partir d'une étude exploratoire qualitative, nos résultats suggèrent que le type de capital social mobilisé explique en partie les motifs de leur utilisation. Par ailleurs, notre analyse met en exergue les motivations sociales, moins souvent explorées dans la littérature.

\section{Informal solidarity finance in Peru from a social capital perspective}

This research explores the reasons for participating in informal solidarity finance in the context of Latin America and, in particular, Peru, from a social capital perspective. We examine the case of rotating savings and credit associations in which lending is based on trust and shared information. Despite the development and consolidation of microfinance in Peru, rotating savings and credit associations are still widely used. Based on an exploratory qualitative study, our findings suggest that the type of social capital employed partly explains the reasons for their use. Our work also highlights the social motivations, which are less often studied in the literature.

\section{Finanzas solidarias informales en Perú desde la perspectiva del capital social}

A través de la noción de capital social, esta investigación explora las razones que explican la participación a ciertas formas de finanzas solidarias informales en el contexto de América Latina, en particular en el caso de Perú. Con este fin, analizamos el caso de las Asociaciones de Ahorro y Crédito Rotativo, una forma de crédito basado sobre la confianza y la información compartida. A pesar del desarrollo y la consolidación de las microfinanzas en este país, se observa que el crédito rotativo informal es aun utilizado. A partir de un estudio exploratorio cualitativo, nuestros resultados sugieren que el tipo de capital social movilizado explica, en parte, las razones de su uso. Por otra parte, el análisis pone en relieve las motivaciones sociales, las cuales son exploradas con menos frecuencia en la literatura. 
Au cours des dernières décennies, le secteur de la microfinance a connu un essor important en Amérique latine (Labie, 2010; 2013). Dans plusieurs pays, les responsables politiques lui ont donné des cadres adaptés à ses besoins (Trujillo Tejada et al., 2013). Au Pérou, les organisations de microfinance ont répondu très efficacement aux besoins des acteurs ayant des difficultés d'accès aux marchés financiers (Aguilar Andia, 2013; Valenzuela Ramirez, 2011). Par ailleurs, une partie du système bancaire a réorienté certaines de ses activités vers les microfinances.

En marge des finances solidaires « formelles » (enregistrées et encadrées par la loi), les systèmes financiers informels (systèmes ou activités légaux, mais qui ne sont pas enregistrés ou encadrés par la loi) ont continué à opérer. Le phénomène a persisté: "Alors qu'on avait longtemps réfléchi en termes de substitution, pensant que la microfinance allait remplacer les pratiques informelles, on sait aujourd'hui qu'il n'en est rien et que, au contraire, elles sont davantage complémentaires que substituables" (Labie, Vanroose, 2013, p. 22). Cette coexistence est expliquée par des raisons économiques (Guirkinger, 2008) ou par la signification sociale de finances informelles (Guérin et al., 2009).

La formalisation croissante et le dynamisme des organisations financières solidaires qui répondent aux besoins de plusieurs acteurs individuels et collectifs, ainsi que la coexistence des systèmes formels et informels, nous amènent à explorer la question suivante: quelles sont les raisons motivant la participation à des finances solidaires informelles dans le contexte péruvien? Nous proposons d'analyser le cas des associations d'épargne et de crédit rotatif $\left(\mathrm{AECR}^{(1)}\right)$, composante d'un système financier

(1) Dans la littérature, ellessont souvent connues sous le nom de Rosca (rotating saving and credit associations). Cependant, la dénomination change selon la région. En Afrique, elles reçoivent les noms de tontine, esusu, tonti, upatu, madras, keneti, toco, susu. En Asie, elles sont appelées hui (Taïwan) et chits (Inde). En Amérique, elles sont connues comme tanda (Mexique), partners (Jamaïque), pasanakus (Bolivie), polla (Chili). Au Pérou, elles reçoivent le nom de pandero ou de junta. informel de type tontinier fondé essentiellement sur la confiance et l'information partagée.

En raison de leurs caractéristiques, nous considérons que ces associations sont essentiellement des réseaux sociaux. En fait, la participation aux AECR dépend de la capacité des acteurs à s'organiser collectivement (Levenson, Besley, 1996) et à maintenir des relations sociales basées sur la confiance et la proximité (Servet, 1996). A partir de ce constat, nous proposons d'établir un lien avec la notion de capital social. En référence à Coleman (1988), celle-ci est définie ici comme une ressource issue des relations interpersonnelles qui facilite certaines actions individuelles et collectives. Le capital social permet donc la création d'un type de réseau formé par des acteurs ayant des caractéristiques plus ou moins homogènes, maintenant des interactions fréquentes et partageant une relation horizontale. Concernant la méthodologie, l'étude est circonscrite au cas péruvien. Malgré la présence et l'importance apparente de ce type d'organisations au Pérou, nous n'avons pas trouvé de travaux abordant systématiquement le sujet. En général, celui-ci a été davantage étudié dans les cas de pays africains et asiatiques, autant pour les AECR mises en place par des ménages (Besley et al., 1993; Calomiris, Rajaraman, 1998; Callier, 1990) que pour celles qui sont organisées par des entreprises (Tello-Rozas, Gauthier, 2012).

Notre recherche exploratoire nourrit une réflexion sur la richesse des pratiques d'économie sociale et solidaire en Amérique latine. Nos résultats 
corroborent la fonction économique des AECR, qui a été amplement étudiée par la littérature en économie et management. Cependant, ils suggèrent aussi que, dans certaines AECR, les membres privilégient les motivations sociales, aspect beaucoup moins traité par cette littérature. L’analyse indique également que certaines pratiques d'économie sociale et solidaire (par exemple, les finances solidaires) acquièrent une signification distincte dans chaque contexte (Defourny, Nyssens, 2010; Guérin et al., 2009; Vaillancourt, 2006), en fonction des caractéristiques, des besoins et des aspirations des acteurs locaux.

\section{Les AECR et le capital social dans la littérature}

\section{Les associations d'épargne et de crédit rotatif}

Les AECR sont des associations financières informelles nées il y a plus d'un siècle. On les retrouve aujourd'hui dans certains pays d'Asie (Levenson, Besley, 1996, Calomiris, Rajaraman, 1998), d’Afrique (Besley et al., 1993; Tello-Rozas, Gauthier, 2012) et d'Amérique (Adams, Canavesi de Sahonero, 1989), de même que parmi certains groupes dans des pays développés (Scholten, 2000; Velez-Ibanez, 1983).

De manière générale, il s'agit d'une "association formée par un groupe de personnes qui acceptent de verser des cotisations à une cagnotte, laquelle est attribuée, complètement ou en partie, à chaque personne à tour de rôle " (Ardener, 1964, p. 201, traduction de l'auteure). Concernant les motifs qui incitent à participer aux AECR, la grande majorité des spécialistes du sujet mettent en relief leur fonction économique. S'inscrivant dans cette perspective, Besley et al. (1993) proposent un modèle qui explique le mécanisme de fonctionnement et les résultats à la fois des AECR par tirage et des AECR par enchère. Leurs résultats suggèrent que les fonds acquis à partir de la participation à une AECR sont entièrement consacrés à l'achat d'un bien indivisible. Sur ce dernier aspect, Calomiris et Rajaraman (1998) présentent une opinion différente. En analysant les AECR par enchère, ils affirment que celles-ci sont utilisées comme mécanismes d'assurance: la cagnotte sert à se protéger des événements imprévus (maladies, désastres naturels, etc.).

La littérature traite donc les AECR comme la réaction des agents économiques aux problèmes d'asymétrie d'information et à l'exclusion du marché financier (Guérin et al., 2009; Baixeras Donoso et al., 2011). En ce sens, les AECR «peuvent être comprises comme la réponse d'un groupe socialement connecté à l'exclusion du marchéde crédit »(Besley et al., 1993, p. 807, traduction de l'auteure).

Etant donné l'importance des liens sociaux pour la création des AECR et leur fonctionnement, couplée au fait que le capital social et non des biens offerts en garantie serve de collatéral aux membres des associations (Bebbington, Gomez, 2006), nous explorons dans la section suivante le corpus de littérature qui porte sur la notion de capital social en lien avec les organisations informelles.

\section{Le capital social}

Le capital social, notion utilisée dans plusieurs analyses contemporaines de l'interaction des individus et des organisations (Kwon, Adler, 2014), est défini ici à partir des postulats de Coleman (1988). Selon ce dernier, 
chaque personne fait partie d'un contexte social particulier et a à sa disposition certaines ressources pour atteindre ses objectifs, parmi lesquelles figure le capital social. Cette ressource s'instaure, se renforce ou se déprécie dans le temps (Bourdieu, 1980; Granovetter, 1983). Woolcock (1998) propose une analyse plus large en indiquant que le capital social peut être envisagé comme la nature et le degré des relations autant personnelles qu'institutionnelles. Cette perspective permet d'étudier le sujet sur deux niveaux. Le premier niveau, dit micro, comprend l'ensemble des relations et des interactions entre les individus d'une communauté (liens intracommunautaires), ainsi que les liens extracommunautaires (entre les individus des divers groupes); le second, dit macro, les relations entre l'Etat et la société civile. Dans la même ligne de pensée, Putman (1994) différencie deux types de capital social: le bonding, qui fait référence à des groupes fondés sur des liens forts, et le bridging, grâce auquel des relations entre différents réseaux s'établissent.

La notion de capital social est également utile pour mesurer l'efficacité et le fonctionnement des organisations informelles. Un premier groupe d'auteurs souligne les qualités de ce type d'organisations: les "institutions non marchandes » basées essentiellement sur le capital social deviennent un élément clé dans le processus de développement (Grief, 1993) et servent à combattre la pauvreté (Woolcock, Narayan, 2000) ainsi qu'à diminuer certains types de risques (Besley, 1995).

Un deuxième groupe d'auteurs souligne au contraire la nécessité d'institutionnaliser ou de formaliser les organisations informelles (Baixeras Donoso et al., 2011; Durlauf, Fafchamp, 2004; Shirley, Keefer, 2000). L'existence d'organisations formelles serait en effet indispensable pour atteindre des niveaux de développement plus élevés.

Compte tenu de cette dernière perspective, nous pourrions supposer que la présence d'institutions formelles fait disparaître, ou au moins réduit, l'action du capital social. Cependant, l'expérience de pays développés suggère que le capital social peut continuer à agir grâce à divers mécanismes (Healy, Côté, 2001) et que les organisations formelles et informelles sont complémentaires (Labie, Vanroose, 2013; Guérin et al., 2009).

En parcourant la vaste littérature relative au capital social, nous constatons que la notion est rarement mobilisée pour analyser la persistance des organisations financières informelles dans des contextes où le marché formel devient plus efficace (comme dans les pays émergents et certains pays en développement). Nous proposons d'utiliser cette notion pour explorer les motivations des membres des AECR dans le contexte péruvien.

\section{Contexte et méthodologie}

La croissance économique soutenue observée au Pérou ( $9 \%$ annuellement en moyenne de 2003 à 2013, selon l'Institut national de statistique du Pérou, INEI, 2015) a conduit à une expansion du marché financier. Le nombre d'institutions bancaires et les dépôts et crédits dans les banques ont augmenté significativement (Valenzuela Ramirez, 2011), parallèlement à l'activité des organisations de microfinances. Selon Aguilar Andia (2013), 
le portefeuilledes prêts chez ces dernières s'est accru à un taux annuel moyen de $30 \%$ entre 2001 et 2009 , et le nombre d'institutions est passé de 240 en 2001 à 1066 en 2009. Les régions aux indices de pauvreté les plus élevés contiennent beaucoup plus d'institutions de microfinance que de banques.

Le secteur péruvien de la microfinance présente certaines défaillances, notamment l'offre massive et la saturation dans certaines régions (Sinclair, 2014). Toutefois, ce pays est considéré comme très propice au développement du microcrédit: le Centre pour l'inclusion financière (Center for financial inclusion, CFI), think tank développant des actions pour une inclusion financière mondiale, classe le Pérou comme le pays offrant le meilleur environnement pour la microfinance depuis plusieurs années et indique qu'il « maintient sa place de leader (dans la microfinance) en démontrant un environnement réglementaire bien équipé, un marchécompétitifet innovant et un leadership sur des mesures de protection du client» (The Economist Intelligence Unit, 2013, p. 10, traduction de l'auteure).

Malgré cette performance, les faits révèlent la coexistence d'institutions formelles de microfinance et de mécanismes informels. Guirkinger (2008), par exemple, constate celle des marchés de crédit formel et informel dans le nord du Pérou. A partir d'analyses économétriques, l'auteure explique que la participation à des mécanismes informels répond à trois objectifs économiques et financiers: surmonter l'exclusion du marché de crédit formel, pour les ménages sans biens à offrir en garantie; réduire les coûts de transaction liés à l'utilisation de mécanismes formels, pour les ménages ayant accès au système bancaire et à des organisations de microcrédit, qui choisiraient de faire appel à des associations et à d'autres mécanismes de crédit et d'épargne non enregistrés pour éviter les coûts liés au temps et aux exigences légales; enfin, diminuer le risque, les ménages utilisant le crédit et l'épargne informels auraient accès à des informations locales qui leur permettraient d'établir des contrats discrétionnaires appropriés à chaque situation précise. Au vu de la persistance des organisations informelles de finance solidaire et de leur coexistence avec des mécanismes formels, il est pertinent d'expliquer davantage la participation aux AECR. Nous nous demandons notamment s'il n'existe pas d'autres causes que les motifs d'ordre économique évoqués généralement dans la littérature.

Quant à la méthodologie utilisée pour explorer ce qui sous-tend la participation aux AECR en contexte péruvien, nous optons pour une démarche qualitative. La principale source de données a été l'entretien: nous en avons réalisé six semi-structurés en identifiant quatre personnes qui, en apparence, ont accès au marché financier, et deux autres sans accès apparent ou avec un accès limité. Considérant que la triangulation de données permet de réduire les risques d'interprétations erronées (Denzin, Lincoln, 2003) et les problèmes de validité des données (Glesne, 1999), les informations obtenues à partir de ces interviews ont été complétées par l'observation participante: nous avons pris part à une soirée de rencontre d'une AECR. Cinq entretiens ont été réalisés avec des personnes habitant le Pérou, et le sixième a été effectué à Montréal. Pour ce dernier cas, nous avons interrogé un immigrant péruvien qui habite au Québec depuis plus de dix ans. 
Conformément à nos critères d'échantillonnage, les personnes interviewées participaient ou avaient participé à des AECR à trois reprises au moins. Par ailleurs, toutes sont adultes, ont des enfants, travaillent ou sont à la retraite; elles perçoivent donc un revenu mensuel.

En ce qui concerne l'analyse des informations collectées, nous nous sommes appuyés sur la grille suggérée par Gioia et al. (1994). La transcription des entretiens a été codée à l'aide du logiciel Atlas.ti et ces codes nous ont servi à identifier des catégories d'analyse.

\section{Motivations et facteurs expliquant la participation aux AECR}

Nous organisons la présentation des résultats à partir des motivations exprimées lors des entretiens, la première étant l'accès aux marchés financiers. Nos interviewés indiquent qu'une des raisons qui les ont incités à participer aux AECR est le coût des services bancaires. Selon eux, il est plus coûteux de confier l'argent à une institution financière que de le maintenir inactif ou de l'utiliser pour participer à des associations plutôt informelles : «...J'ai sorti mon argent de la banque, parce que le taux d'intérêt que les banques paient ici au Pérou est plus petit que les taux qu'elles chargent pour la manutention [du compte]... tu perds» (entretien 3$)$.

L'entretien avec le Péruvien habitant le Canada suggère également que, dans un contexte où les marchés financiers sont bien développés, les AECR seraient une possibilité d'intégration pour certains groupes d'immigrants: "Les gens ne connaissent pas [le système]..., ils ne savent pas parler, par exemple. Pour eux, les personnes de la banque ne donnent pas d'information, ils sont un peu fermés aussi. La banque a tous les services pour tout le monde, mais ils ne le disent pas. Si tu sais, tu demandes, mais sinon tu ne demandes pas. Et encore, lorsque tu poses des questions, les employés de la banque te répondent de la mauvaise manière. Donc, tu n'y retournes pas, n'est-ce pas?» (entretien 2).

Par ailleurs, comme dans les citations précédentes, nos informateurs font ressortir l'utilisation des AECR comme moyen d'épargne: "La plupart du temps, c'est pour aider à réaliser des choses qui ne peuvent pas se faire dans un moment donné, [la participation aux AECR] vous oblige à épargner» (entretien 2). En dehors de ces raisons, l'analyse des entretiens suggère deux autres facteurs expliquant la participation aux AECR : avoir accès à des fonds additionnels et pouvoir participer à une activité sociale. Les informations recueillies nous montrent que deux de nos informateurs participent ou ont participé aux AECR pour le premier motif, soit un besoin économique. Voici comment ils décrivent leur intérêt pour ces associations: «Bon, la chose la plus intéressante est quand tu vas recevoir ton petit argent, bien sûr ma fille, lorsqu'on va recevoir on est anxieux, on se dit: tel jour est mon tour, il manque telle quantité de jours, untel a déjà reçu, il ne manque que deux personnes, et ainsi jusqu'à avoir ton tour. On est comme ça» (entretien 1$)$.

"[L'AECR] t'aide à accomplir tes objectifs, à acheter ce que tu veux, c'est ça, ou à accomplir tes rêves, à réaliser un voyage, n'est-ce pas? Avec tes 5000 dollars, tu peux aller dans une croisière, les gens, ils le font » (entretien 2 ). 
Les personnes qui attribuent leur participation au caractère social des AECR donnent des explications clairement différentes : "Qu'est-ce que tu aimes le plus des AECR, ce que tu trouves plus intéressant? - Plus... la relation d'amitié» (entretien 3).

"Ce que j’aime le plus... c'est de se rencontrer avec les amis. Avec ce prétexte, on se voit au moins une fois par mois» (entretien 5).

En considérant ces deux facteurs, nous allons présenter quatre thèmes des AECR qui se dégagent des entretiens : les caractéristiques des membres et les liens entre eux, la taille du groupe, la forme d'organisation, la périodicité de l'attribution de la cagnotte et celle des rencontres.

\section{Caractéristiques des membres et liens entre eux}

Parmi les descriptions des six entretiens, nous constatons qu'un des groupes était constitué exclusivement de femmes, mais l'interviewée n'indique pas que le genre soit une sorte de filtre pour choisir les participants. Nous remarquons par ailleurs que les groupes peuvent être hétérogènes en ce qui concerne le sexe et l'âge des participants. L'un de nos informateurs révèle par exemple qu'il y a «toutes sortes de personnes. Dans cette AECR, ily avait une collègue du travail, puis une dame de 60 ans, un peu de tout » (entretien 6). Cependant, en procédant à une analyse détaillée des informations, on constate que l'information partagée est un élément clé pour la constitution d'un tel type d'association. Il s'agit de collègues de travail, d'anciens camarades d'école, de gens partageant une même dévotion, ou venant du même pays. Les six personnes interrogées soulignent que, pour faire partie d'une AECR, les membres doivent nécessairement se connaître : "Bon, tu dois connaître la personne. Elle doit être une amie ou une collègue de travail que tu vois continuellement, n'estce pas? Ce n'est pas tout le monde qui peut [en faire partie] »(entretien 1). Par ailleurs, ceux qui utilisent l'AECR comme activité sociale suggèrent que les liens avec les autres participants sont très étroits, il s'agit ici de liens d'amitié ou de liens familiaux. "Normalement, ceux qui font une AECR, ce sont des amis, des parents, des amis de l'école aussi »(entretien 6).

Ce sont ces liens et l'information partagée entre les membres qui diminuent les risques de défaut : "C'est comme si vous payeriez une dette à la banque. Tout le monde te connaît et les gens qui obtiennent ces AECR doivent être responsables, comme si elles devaient payer les factures d'une banque» (entretien 1). En cas de fraude, les mécanismes de renforcement sont principalement sociaux : nos informations laissent entendre que les sanctions ne sont pas d'ordre légal, mais plutôt de nature sociale : "Dans ce cas, ils cherchent toutes les manières pour te faire payer. J'ai connu un tel cas. Oui [une personne qui n'a pas payé sa contribution], ils lui ont demandé, et finalement ils ont décidé d'arrêter de lui parler » (entretien 4).

Il est pertinent de souligner que les interviewés montrant une motivation sociale affirment que, dans les AECR basées sur des liens étroits, les participants apportent leur contribution à coup sûr, la probabilité de défaut devient donc presque nulle : "Tous doivent y donner. C'est un engagement qui est fait au début. Personne ne peut manquer» (entretien 5). 


\section{Taille du groupe et forme d'organisation}

Nous avons identifié certaines informations cohérentes avec les faits discutés dans la littérature. Dans la plupart des cas, il s'agit de groupes relativement petits qui varient entre sept et vingt membres. Une seule personne parmi les six questionnées indique avoir participé à une AECR de quarante personnes. Nous avons également remarqué que le nombre de participants dépend des liens entre les membres de l'AECR et de leurs motivations. Ainsi, les groupes où le lien entre les individus est étroit (amis très proches) et dans lesquels la participation poursuit surtout un objectif social sont plus petits (de sept à douze membres).

Les informations recueillies suggèrent de plus certaines différences quant à la forme d'organisation. Nous observons que les éléments permettant la réalisation de chaque AECR (coordination, responsables, responsabilités, etc.) sont aussi reliés aux objectifs de participation. Les interviewés qui montrent un intérêt principalement économique indiquent que la personne qui organise l'AECR a plusieurs responsabilités. Elle détermine la liste des participants (en prenant en compte l'information disponible sur chaque personne), invite les personnes à participer, établit le montant de la cagnotte et la périodicité de chaque contribution, contrôle la distribution de cette cagnotte à chaque période et, ce qui est plus important, assume entièrement la responsabilité en cas de défaut. En contrepartie, l'organisateur a l'avantage de recevoir la première cagnotte.

En revanche, dans les cas où l'AECR sert d'activité de rencontre, nous constatons qu'il n'y a pas un organisateur unique. La coordination est faite par chacun des membres à tour de rôle, c'est-à-dire que tous les participants partagent les responsabilités. Le rôle de l'organisateur est, dans ce cas, rotatif et consiste à déterminer l'endroit pour la rencontre (un café, un restaurant, au domicile d'un membre) et les activités à réaliser (un cocktail, un souper). Nos interviewés précisent, par exemple: «Parfois elles proposent ici ou là-bas, généralement, nous nous rejoignons dans un restaurant, pas trop grand pour avoir plus de tranquillité, pour que personne ne nous presse» (entretien 5). "Regarde par exemple, l'AECR avec les $X$ on le fait avec les enfants. Donc, nous nous rejoignons chez quelqu'un, on discute, on mange, on parle, on rit, tout dans une ambiance plutôt amicale, puis ils partent tôt [...]. Mais ily a des AECR dans lesquelles, disons, les gens se rejoignent chez quelqu'un, on prend quelque chose à boire et donc ils vont danser, toujours, tout le monde, tout le grand groupe»(entretien 4 ).

\section{Périodicité de l'attribution de la cagnotte et celle des rencontres}

La périodicité de l'attribution de la cagnotte suit la même logique. Elle varie selon les intérêts des membres du groupe. L’information collectée montre que les gens avec un intérêt économique participent aux AECR dont la rotation est plus rapide (chaque semaine, ou tous les quinze jours). Cependant, un élément commun est souligné dans tous les entretiens : les dates de contribution et d'attribution coïncident avec celles auxquelles les gens reçoivent leur salaire. Par ailleurs, la participation aux AECR est limitée aux individus qui ont la capacité économique de faire une contribution périodique. 
Quant à la périodicité des rencontres, elle varie notablement parmi les interviewés. Dans le cas des groupes qui trouvent dans les AECR une occasion pour se rejoindre et participer à une activité sociale, les membres du groupe se réunissent chaque fois que la cagnotte est attribuée, par exemple une fois par mois. Ainsi, l'AECR devient une activité de loisir. Dans les autres cas, les groupes réalisent une seule réunion dans laquelle les règles de participation sont établies. Par la suite, c'est l'organisateur qui contacte chaque membre individuellement, afin de lui demander sa contribution et de distribuer la cagnotte.

Pour ce qui est des contributions, tous nos informateurs déclarent avoir toujours participé à des AECR avec des contributions fixes dans le temps, c'est-à-dire que le montant était le même pendant toute la durée de l'AECR. Nous constatons aussi que trois des cinq personnes interviewées au Pérou effectuent leurs contributions en monnaie étrangère (dollars américains ou euros), se protégeant, de cette manière, des fluctuations de la monnaie nationale. Elles indiquent aussi que l'attribution de la cagnotte se réalise presque toujours par tirage au sort. Un seul informateur mentionne avoir participé à une AECR où l'ordre d'attribution était décidé par un accord volontaire de tous les membres.

Il convient de remarquer un dernier élément dans la description des AECR : la flexibilité que comportent ces associations. Bien que tous les participants assument la responsabilité de contribuer périodiquement, il peut se produire un bref retard dans la date de la contribution. De même, ils peuvent négocier la date d'attribution de la cagnotte avec les autres membres pour répondre à des besoins urgents.

\section{Discussion et conclusions}

La littérature s'intéressant aux AECR sous l'angle de l'économie et du management indique que les bénéficiaires de ce type de finances solidaires informelles y participent pour les raisons suivantes : ils n'ont pas accès (ou alors un accès limité) aux marchés de crédit (Besley et al., 1993) ; les marchés de capitaux sont absents (Scholten, 2000) ; les coûts de transaction des finances formelles sont élevés ; les ménages ayant des revenus assurés (Baixeras Donoso et al., 2011) cherchent à diminuer les risques liés aux asymétries d'information (Guirkinger, 2008). L'analyse de nos résultats corrobore plusieurs de ces constats : la participation aux AECR relève de motivations économiques et financières. Cependant, un aspect souvent négligé dans cette littérature apparaît : outre les motivations économiques, certaines personnes font partie des AECR pour des motivations sociales. Plusieurs de nos interviewés font ressortir d'abord leur désir d'établir ou de renforcer leurs liens avec des amis grâce à leur participation aux AECR.

Certains éléments caractérisant les AECR tels que l'information partagée, les relations horizontales entre les membres et la présence de sanctions sociales justifient l'utilisation de la notion de capital social. Cette dernière permet de dégager des pistes de réponses à notre question de recherche : quelles sont les raisons qui motivent la participation à des finances solidaires informelles dans le contexte péruvien? 
L'analyse des données qualitatives nous permet de constater que le rôle des AECR varie selon les besoins du groupe qui les met en place. En considérant les caractéristiques de ces associations, celles des participants ainsi que la forme de capital social qu'ils partagent, nous avons identifié deux groupes différents. Le premier est caractérisé par la présence de liens sociaux larges entre les membres (capital social de type bridging). Nos résultats suggèrent que, pour ce groupe, les AECR ont deux rôles: elles servent à financer la consommation, à épargner des fonds pour une consommation future et à surmonter l'absence d'un historique de crédit. Ce constat est cohérent avec la littérature attribuant l'existence de ce type d'organisations financières informelles aux défaillances du système formel (Calomiris, Rajaraman, 1998; Besley et al., 1993). Parallèlement, l'association donne la possibilité à ses membres d'interagir avec d'autres personnes ayant des caractéristiques similaires (par exemple des immigrants du même pays), d'établir des liens et, par conséquent, de construire un capital social.

Les caractéristiques et motivations évoquées par le deuxième groupe sont plutôt contre-intuitives au regard des constats de la littérature économique et managériale sur les AECR. En analysant les AECR mises en place par des groupes aux liens sociaux très étroits (capital social du type bonding), nous apprenons plutôt que la participation à ces associations cherche principalement à renforcer les liens sociaux entre les membres du groupe. Les interviewés formant ce deuxième groupe font ressortir le rôle économique des AECR, mais ils soulignent surtout leur fonction sociale.

Le tableau 1 résume les caractéristiques des AECR auxquelles participent nos informateurs et les objectifs de leur participation à partir du type de capital social mobilisé. Les caractéristiques différenciées des deux groupes identifiés laissent à penser que les motivations des membres des AECR sont doubles: économiques et sociales.

Certains auteurs se sont interrogés sur les raisons expliquant la coexistence actuelle des marchés financiers formels et informels en Amérique latine (Guirkinger, 2008; Baixeras Donoso et al., 2011). Nos travaux suggèrent que les AECR ont une double fonction, au moins dans le contexte de certains pays en développement ayant réussi à renforcer leurs marchés financiers: elles permettent de surmonter les défaillances du marché financier pour les personnes n'ayant pas d'historique de crédit, d'accéder à des ressources financières et d'épargner des fonds pour financer la consommation future. Parallèlement, les AECR font office de mécanismes de renforcement des liens sociaux. Cette caractéristique pourrait expliquer, en partie, la coexistence ci-dessus mentionnée. Enfin, l'étude montre que la participation aux AECR servirait à renforcer le capital social qui, selon Bourdieu (1980) et Granovetter (1983), peut se déprécier dans le temps.

A partir des résultats de cette étude exploratoire, nous pourrions émettre des hypothèses quant à l'utilisation que les classes moyennes font des finances formelles et informelles dans le contexte des pays en développement, en particulier en Amérique latine: par leur fonction sociale, les finances informelles ne se substitueraient pas aux mécanismes formels, elles seraient plutôt complémentaires. Par ailleurs, la relation entre type d'objectif, social ou économique, et les différentes caractéristiques observées (la taille 


\section{Tableau 1}

Comparaison des caractéristiques des deux types de groupes identifiés selon le capital social mobilisé.

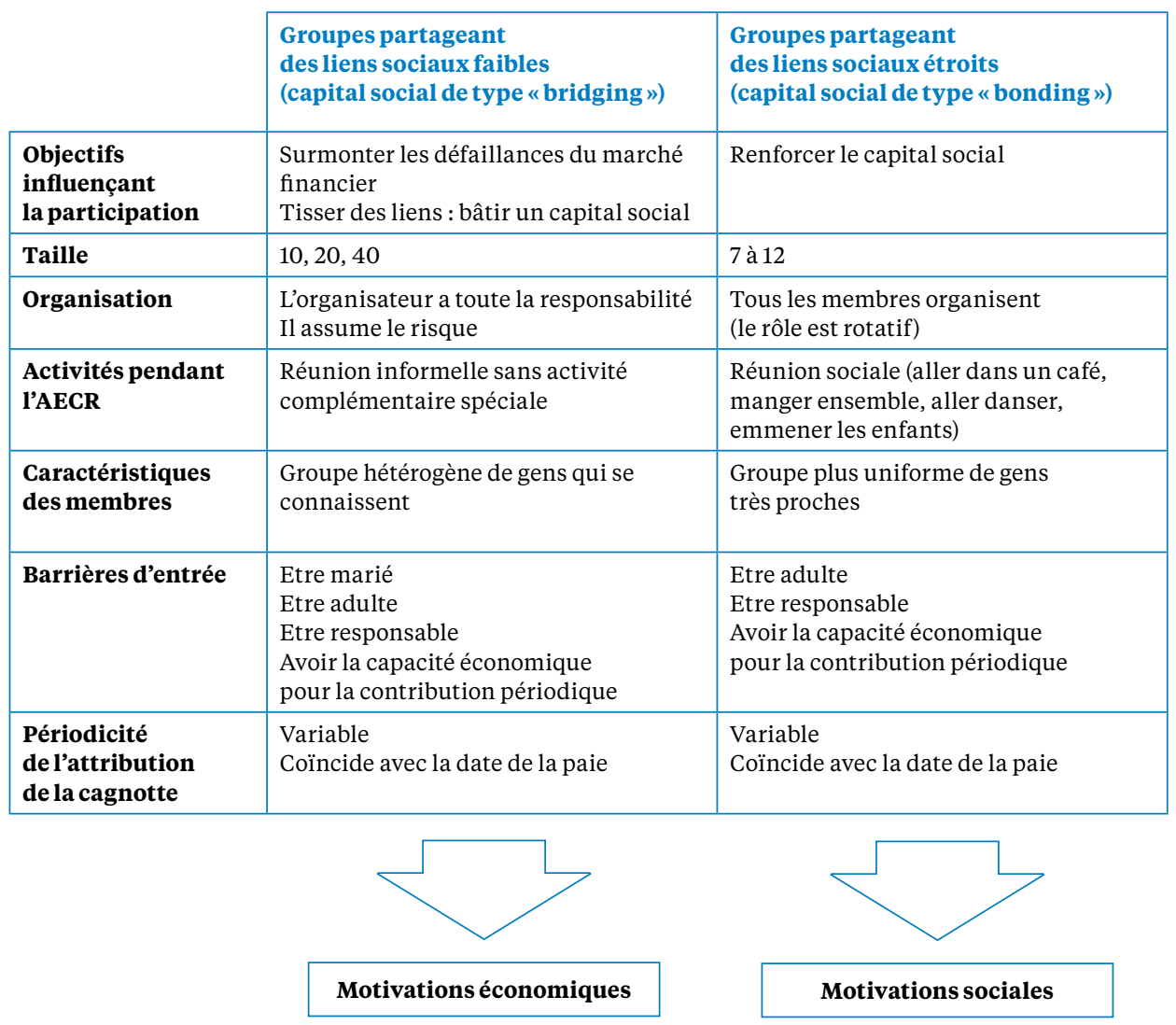

du groupe, le partage du risque, etc.) deviennent un élément intéressant à tester dans de futures recherches.

A la suite de Defourny et Nyssens (2010) et Vaillancourt (2006), nous pensons que les pratiques des finances solidaires, des microfinances informelles et de l'économie sociale et solidaire en général répondent aux caractéristiques et aux besoins de chaque contexte. Dans le cas des microfinances, si, pour un groupe, elles servent principalement de mécanisme d'accès à la consommation et aux services financiers, pour d'autres, les AECR constituent une manière de bâtir et d'entretenir le capital social. Nos résultats nourrissent ainsi la discussion sur la diversité des formes de la finance solidaire en Amérique latine. Les limites de la recherche doivent, néanmoins, être relevées. Il s'agit d'une étude exploratoire reposant sur un nombre limité d'entretiens (six) et sur une observation participante. La comparaison avec les informations issues d'un autre contexte (par exemple un autre pays d'Amérique latine) et la vérification de nos résultats grâce à des données complémentaires pourrait enrichir, valider et compléter notre analyse. 


\section{BiBLIOGRAPHIE}

Adams D., Canavesi de Sahonero M., 1989, "Rotating savings and credit associations in Bolivia ", Savings and development, XIII, p. 219235.

Aguilar Andia G., 2013, " Microfinanzas y crecimiento regional en el Perú ", Economía, vol. XXXVI(72), p. 143-173.

Ardener S., 1964, « The comparative study of rotating credit associations ", Journal of the royal antropological institute of Great Britain and Ireland, $\mathrm{n}^{\circ}$ 94, p. 201-229.

Baixeras Donoso S., Altunbaş Y., Kara A., 2011, « The rationale behind informal finance: Evidence from Roscas in Bolivia », The journal of developing areas, vol. 45(1), p. 191-208.

Bebbington D., Gomez A., 2006, « Rebuilding social capital in post-conflict regions: women's village banking in Ayacucho, Peru and in highland Guatelama ", in Fernando J. (éd.), Microfinance: Perils and prospects, Routledge, New York, p. 97-114.

Besley T., Coate S., Laury G., 1993, « The economics of rotating saving and credit associations ", The American economic review, $\mathrm{n}^{\circ} 83$, p. 792-810.

Besley T., 1995, " Nonmarket institutions for credit and risk sharing in low-income countries", Thejournal of economic perspectives, $\mathrm{n}^{\circ}$ 9, p. 115-127.

Bourdieu P., 1980, « Le capital social », Actes de la recherche en sciences sociales, $\mathrm{n}^{\circ} 23, \mathrm{p} .31$.

Callier P., 1990, «The informal finance, role of Roscas: The rotating saving and credit associations, An interpretation ", Kyklos, $\mathrm{n}^{\circ} 43$, p. 273-276.

Calomiris C., Rajaraman I., 1998, « The role of Roscas: Lumpy durables or event insurance? ", Journal of development economics, $\mathrm{n}^{\circ} 56$, p. 207-216.

Coleman J., 1988, «Social capital in the creation of human capital ", The American journal of sociology, ${ }^{\circ}$ 94, supplément : "Organizations and institutions: Sociological and economic approaches to the analysis of social structure ", p. S95-S120.
Defourny J., Nyssens M., 2010, « Conceptions of social enterprise and social entrepreneurship in Europe and the United States: Convergences and divergences ", Journal of social entrepreneurship, vol.1(1), p. 32-53.

Denzin N., Lincoln Y., 2003, Strategies of qualitative inquiry, Thousand Oaks, California Sage. DurlaufS.,Fafchamps M., 2004, Socialcapital, Cambridge, MA, NBER Working Paper Series, $\mathrm{n}^{\circ} 10485$.

Gioia D., Thomas J., Clarck S., Chittipeddi K., 1994, "Symbolism and strategic change in academia: The dynamics of sensemaking and influence », Organization science, $n^{\circ} 5$, p. 363-383. Glesne C., 1999, Becoming qualitative researchers, New York, Longman.

Granovetter M., 1983, « The strength of weak ties: A network theory revisited ", Sociological theory, ${ }^{\circ} 1$, p. 201-233.

Grief A., 1993. " Contract enforceability and economic institutions in early trade: Maghribi trader's coalition ", The American economic review, $\mathrm{n}^{\circ} 83$, p. 525-548.

Guérin I., Morvant-Roux S. et Servet J.-M., 2009, Understanding the diversity and complexity of demand for microfinance services: Lessons from informal finance, Rume Working Papers Series, 2009-7.

Guirkinger C., 2008, " Understanding the coexistence of formal and informal credit markets in Piura, Peru ", World development, vol. 36(8), p. 1446-1452.

Healy T., CôtéS.,2001, Du bien-être des nations: le rôle du capital humain et social, Paris, Organisation de coopération et de développement économiques (OCDE).

INEI, 2015, Principales indicadores macroeconómicos. Inei.gob.pe/estadisticas/indicetematico/economia (consulté le 9 février 2015).

Kwon S. W., Adler P., 2014, "Social capital: Maturation of a field of research ", Academy of management review, vol. 39(4), p. 412-422. Labie M., 2010, « Microfinance : le temps de la maturité ? ", Mondes en développement, vol. 152(94), p. 711. 
Labie M., 2013, «Introduction. La microfinance latino-américaine : entre commercialisation et nouvelles gauches ", Mondes en développement, vol. 163(3), p. 7-8.

Labie M., Vanroose A., 2013, « La légitimité en microfinance : tentative d'application de la théorie du champ au cas latino-américain ", Mondes en développement, vol. 163(3), p. 21-36.

Levenson A., Besley T., 1996, « The anatomy of an informal financial market: Rosca participation in Taiwan ", Journal of development economics, $\mathrm{n}^{\circ}$ 51, p. 45-68.

Putnam R.,1994, Makingdemocracywork: Civic traditionsin modern Italy, New Jersey, Princeton University Press.

Scholten U., 2000, « Rotating savings and credit associations in developed countries: The German-Austrian Bausparkassen ", Journal of comparative economics, $\mathrm{n}^{\circ} 28$, p. 340-363.

Servet J.-M., 1996, « Risque, incertitude et financement de proximité en Afrique : une approche socio-économique ", Tiers Monde, tome 73(145), p. 41-57.

Shirley M., Keefer P., 2000, Formal versus informal institutions, Cambridge University Press.

Sinclair H., 2014, Trouble brewing in Peru? MiBanco in Dire Straits, Blog.microfinance transparency.com/trouble-brewing-in-perumibanco-in-dire-trouble.

Tello Rozas S., Gauthier B., 2012, " Les tontines favorisent-elles la performance des entreprises au Cameroun? ", Revue d'économie du développement, vol. 26(1), p. 5-39.
The economist intelligence unit, 2013, Global microscope on the microfinance business environnement 2013.

Trujillo Tejada V., Patino V. M., Rodriguez Lopez F., 2013, « La microfinance en Amérique latine : existe-t-il des cadres types de régulation? », Mondes en développement, vol.163(3), p. 37-50.

Vaillancourt Y., 2006, "Le tiers secteur au Canada : un lieu de rencontre entre la tradition américaine et la tradition européenne ", Canadian review of social policy (Revue canadienne de politique sociale), $\mathrm{n}^{\circ} 56$, p. 23-39. Valenzuela Ramirez I., 2011, « Profundidad de alcance en un contexto de comercialización de las micro finanzas en el Perú ", Economía, vol. XXXIV(68), p. 33-63.

Velez-Ibanez C., 1983, Bonds of mutual trust: The cultural systems of rotating credit associations among urban Mexicans and Chicanos, Nouveau-Brunswick, New Jersey. Rutgers University Press.

Woolcock M., 1998, « Social capital and economic development: Toward a theoretical synthesis and policy framework ", Theory and society, $\mathrm{n}^{\circ} 27, \mathrm{p} .151-208$.

Woolcock M., D. Narayan, 2000, "Social capital: Implications for development theory, research and policy ", The world bank research observer, vol. 15(2), p. 225-249. 